\title{
Molecular cloning and expression analysis of the ethylene insensitive 3 (EIN3) gene in cucumber (Cucumis sativus)
}

\author{
B.B. Bie, J.S. Pan, H.L. He, X.Q. Yang, J.L. Zhao and R. Cai \\ Plant Science Department, School of Agriculture and Biology, \\ Shanghai Jiaotong University, Shanghai, China \\ Corresponding author: R. Cai \\ E-mail: cairun@sjtu.edu.cn
}

Genet. Mol. Res. 12 (4): 4179-4191 (2013)

Received September 18, 2012

Accepted January 25, 2013

Published October 7, 2013

DOI http://dx.doi.org/10.4238/2013.October.7.4

\begin{abstract}
The plant gaseous hormone ethylene regulates many aspects of plant growth, development, and responses to the environment. Ethylene insensitive3 (EIN3) is a key transcription factor involved in the ethylene signal transduction pathway. To gain a better understanding of this particular pathway in cucumber, the fulllength cDNA encoding EIN3 (designated as CsEIN3) was cloned from cucumber for the first time by rapid amplification of cDNA ends. The full length of CsEIN3 was $2560 \mathrm{bp}$, with an open reading frame of 1908 bp encoding 635 amino acids. Sequence alignment and phylogenetic analyses revealed that CsEIN3 has high homology with other plant EIN3/EIL proteins that were derived from a common ancestor during evolution, and CsEIN3 was grouped into a cluster along with melon. Homology modeling demonstrated that CSEIN3 has a highly similar structure to the specific DNA-binding domain contained in EIN3/EIL proteins. Based on quantitative reverse transcription-polymerase chain reaction analysis, we found that $C s E I N 3$ was constitutively expressed in all organs examined, and was increased during flower development and maturation in both male and female flowers. Our results suggest that
\end{abstract}


CsEIN3 is involved in processes of flower development. In conclusion, this study will provide the basis for further study on the role of EIN3 in relevant biological processes of cucumber and on the molecular mechanism of the cucumber ethylene signaling pathway.

Key words: Cucumis sativus (cucumber); Expression patterns; Ethylene insensitive3 (EIN3); RACE

\section{INTRODUCTION}

Ethylene $\left(\mathrm{C}_{2} \mathrm{H}_{4}\right)$ was the first example of a gaseous signaling molecule in biological systems, discovered more than a century ago (Neljubov, 1901). It regulates a variety of developmental and stress responses in plants, including seed germination, cell elongation, cell fate, sex determination, fruit ripening, flower senescence, leaf abscission, defense against pathogens, and responses to mechanical trauma, cold, and salt stresses (Lin et al., 2009). Components of the pathway for ethylene signal transduction were identified by genetic approaches in Arabidopsis, and a basic model of the ethylene signal transduction pathway has been established (Guo and Ecker, 2004).

The ethylene insensitive3/ethylene insensitive3-like (EIN3/EIL) proteins, encoded by a small multigene family, are positive regulators of the ethylene signaling pathway at the downstream position that act as transactivating factors to trigger ethylene responses, mainly via the regulation of ethylene response factor $(E R F)$ genes, known to be their downstream targets. Functional domains of EIN3/EIL proteins have been determined by nuclear magnetic resonance spectroscopy. The DNA-binding domains (DBDs), a V-shaped cleft formed by five $\alpha$-helices, were identified from AtEIL3 (Arabidopsis thaliana EIL3). This conserved DBD is also found in all EIN3 homologs examined so far in other plant species. The DBD of EIN3/ EIL binds specifically to the EIN3-binding site located in the promoter region of ethyleneregulated genes (Yamasaki et al., 2005). Some members of EIN3/EIL are regulated at the posttranscriptional level by proteasomal degradation of EIN3 Binding F-Box 1 and F-Box 2 (EBF1 and $E B F 2$ ) that can precipitate the degradation of EIN3/EIL proteins when ethylene is present; in contrast, exogenous ethylene does not affect the accumulation of EIN3/EIL mRNAs (An et al., 2010). However, some EIN3/EIL mRNAs can be regulated at the transcriptional level, based on the fact that exogenous ethylene can regulate the accumulation of mRNA (De Paepe et al., 2004; Parra-Lobato and Gomez-Jimenez, 2011).

As a key nuclear transcription factor in the ethylene signaling pathway, EIN3/EIL proteins have received more attention than other components in recent years. The previous studies indicate that EIN3/EIL proteins can affect the "triple response" of seedlings (Chao et al., 1997), leaf, and root growth (Chao et al., 1997; Lee and Kim, 2003), female flower development (Chao et al., 1997; Hibi et al., 2007), and fruit ripening (Tieman et al., 2001; Chen et al., 2004), and participate in the response to stresses involving salt (Cao et al., 2007; Zhang et al., 2011), cold (Shi et al., 2012), iron deficiency (Bauer and Blondet, 2011), and sulfur deficiency (Maruyama-Nakashita et al., 2006). In addition, EIN3/EIL proteins participate in the cross-talk between ethylene signaling and other signaling, including those of gibberellins (An et al., 2012), salicylic acid (Chen et al., 2009) jasmonic acid (Zhu et al., 2011), and glucose (Yanagisawa et al., 2003). 
Cucumber is an economically important crop as well as a model system for sex determination studies, owing to its rich diversity of sex expression (Huang et al., 2009). Ethylene has been demonstrated to promote female flower development and is highly correlated with femaleness in cucumber (Rudich et al., 1972). To date, some genes that determine the ratio of male-to-female flowers in cucumber have been cloned and they all encode 1-aminocyclopropane-1-carboxylic acid synthase (ACS), a key rate-limiting enzyme in ethylene biosynthesis (Knopf and Trebitsh, 2006; Li et al., 2009). In contrast, still little is known about the role of ethylene signaling in the sex determination of flowers, mainly because the important components in the ethylene signaling pathway have not yet been separated and identified. Only a few components have been isolated from cucumber, including ETR2, ETR1, ERS (Yamasaki et al., 2000), and ERAF17 (Ando et al., 2001), and the majority of key components are still waiting to be isolated and characterized.

Previous studies have discovered that the $M$ locus, a genetic locus that predominantly controls sex type in cucumber, co-segregates with an EIL genomic sequence (Liu et al., 2008). Recently, the $M$ gene was cloned and found to encode ACS (Li et al., 2009). To better understand whether EIN3/EIL proteins act as key transcription factors in the ethylene signal transduction pathway and are indeed involved in sex determination and other biological processes in cucumber, it is imperative to isolate the full-length sequence of EIN3/EIL from cucumber. In the present study, we first isolated the full-length cDNA of EIN3 from cucumber using rapid amplification of cDNA ends (RACE) and then investigated its expression pattern in various cucumber organs and in different developmental stages of both male and female flowers. This work laid the groundwork for further study of EIN3/EIL functions in cucumber.

\section{MATERIAL AND METHODS}

\section{Plant materials and sampling}

The monoecious plant line S52 (inbred line derived from a southern Chinese local cultivar) was used in all the experiments reported in this paper, and was provided by the $\mathrm{Cu}$ cumber Research Group, School of Agriculture and Biology, Shanghai Jiaotong University, China. Plants were grown in a greenhouse at Shanghai Jiaotong University. When the plants had reached the 15 to 20 nodes stage, the taproot and branch root, main stems, mature leaves, shoot apexes, and mature male flowers (MMF) and mature female flowers (MFF) on the day of anthesis, as well as both male and female flowers at five different developmental stages separated on the basis of the corolla length, were collected for the gene expression analysis. With regard to the flower samples, the whole body of the flower, including the calyx, ovary, stamen, pistil, and petal, was collected.

\section{Isolation of RNA and synthesis of the first-strand cDNA}

Total RNA was extracted from material previously frozen in liquid nitrogen and stored at $-80^{\circ} \mathrm{C}$, by using the TRIpure reagent (Aidlab, China) according to the kit instructions. After determining the quality and concentration of RNAs by spectrophotometry for the ratio of $\mathrm{OD}_{260} / \mathrm{OD}_{280}$ and gel electrophoresis, total RNA $(1 \mu \mathrm{g})$ derived from leaves of cucumber $\mathrm{cv}$. S52 was used for the synthesis of the first strand of cDNA according to the manual of the Smart ${ }^{\mathrm{TM}}$ Race cDNA Amplification Kit (Clontech, USA). 


\section{Cloning of full-length cDNA of CsEIN3 and RACE-polymerase chain reaction (PCR) experiments}

A BLASTP search, using the amino acid sequence of the EIN3 gene from Arabidopsis thaliana (GenBank accession No. AEE76421), against the protein database of the Cucumber Genome DataBase (http://cucumber.genomics.org.cn) was conducted, and a tentative consensus sequence of csa012885 located at chromosome 1 was identified. The csa012885 sequence showed $76 \%$ amino acid identity to AEE76421, with an open reading frame (ORF) length of 1395 bp encoding 464 amino acids. Based on the nucleotide sequence of csa012885, a pair of primers covering the cDNA of csa012885 was designed to obtain the cDNA sequence containing the ORF (see Table 1).

\begin{tabular}{lll}
\multicolumn{2}{l}{ Table 1. Primers used in this study. } & \\
\hline Name & Sequence (5'-3') & Utilization \\
\hline CsEIN3-ORF-Fw & TCTGCTGATTCATTTTTGGG & ORF cloning primers \\
CsEIN3-ORF-Re & CGGAAAGAATACGTCGATAAAG & \\
3'RACE-F & CGCCCAAGTCAAATCCACCTCC & RACE primers \\
5'RACE-R1 & GGAAGACAAAGGCGGGCAAGAATCAGG & \\
5'RACE-R2 & CGATCCCTTCCTTAACCTTGCTTTGCTC & Universal primers \\
UPM702 & CTAATACGACTCACTATAGGGCGTACGGCAGCTA & \\
& CAACTCGACAGCTAATACGACTCACTATAGGGC & Full-length cDNA cloning primers \\
UPM & CTAATACGACTCACTATAGGGCAAGCAGTGGTAT & Q-PCR for CsEIN3 \\
NUPA & CAACGCAGAGTCTAATACGACTCACTATAGGGC & \\
CsEIN3-Full-Fw & AAGCAGTGGTATCAACGCAGAGT & Reference gene \\
CsEIN3-Full-Re & GGGTTTTGCTATGAAATTTATGTT & \\
EIN3-Q-2F & TGGATATATAATCAACAACAGGGCA & \\
EIN3-Q-2R & TCCTGATTCTTGCCCGCCT & \\
CsActin3-Fw & GGTTGGTTTCCTCTTTCGCATA & \\
CsActin3-Re & TCGTGCTGGATTCTGGTG & \\
\hline
\end{tabular}

The cDNA sequence containing the ORF of CsEIN3 was amplified in a PCR reaction of 50- $\mu \mathrm{L}$ total volume, containing $40 \mathrm{ng}$ cDNA, $0.5 \mu \mathrm{M}$ primers CsEIN3-ORF-Fwi and CsEIN3-ORF-Re (Table 1), and $25 \mu \mathrm{L} 2 \mathrm{X}$ GoldStar Best MasterMix (CWBiotech, China). The amplification conditions were as follows: $10 \mathrm{~min}$ at $95^{\circ} \mathrm{C}, 30$ cycles of $30 \mathrm{~s}$ at $95^{\circ} \mathrm{C}, 30$ $\mathrm{s}$ at $60^{\circ} \mathrm{C}$, and $2 \mathrm{~min} 50 \mathrm{~s}$ at $72^{\circ} \mathrm{C}$, and a final $5 \mathrm{~min}$ at $72^{\circ} \mathrm{C}$. The amplified products were analyzed by gel electrophoresis on a $1 \%$ gel and recovered with a gel extraction kit (Sangon, China). The recovered product was cloned into the pMD18-T vector (TaKaRa, China) by the method described in the instruction manual and transformed into Escherichia coli strain DH5 $\alpha$, followed by sequencing. The PCR amplification and sequencing of CsEIN3 were repeated three times to avoid any PCR errors. The sequence from this article has been deposited at GenBank under the accession number JQ742004.

Then, the $5^{\prime}$ and $3^{\prime}$ ends of CsEIN3 were obtained by RACE-PCR with the Smart ${ }^{\mathrm{TM}}$ Race cDNA Amplification Kit (Clontech), and CSEIN3 sequence obtained previously was used to design the gene-specific primers (Table 1). The 3' RACE reaction was carried out with primers 3' RACE-F and UPM702 (Universal Primer A Mix), with the following amplification conditions: $5 \mathrm{~min}$ at $94^{\circ} \mathrm{C}$; followed by 30 cycles of $30 \mathrm{~s}$ at $94^{\circ} \mathrm{C}, 30 \mathrm{~s}$ at $65^{\circ} \mathrm{C}$, and $3 \mathrm{~min}$ at $72^{\circ} \mathrm{C}$; and a final extension at $5 \mathrm{~min}$ at $72^{\circ} \mathrm{C}$. Two $5^{\prime}$ gene-specific primers were de- 
signed for the 5' RACE. For the first cycle of amplification of the 5' end of the CSEIN3 cDNA, primers 5' RACE-R1 and UPM702 (Universal Primer A Mix) were used, with the 5' RACEready cDNA as template. For the nested amplification of 5' RACE, primers 5' RACE-R2 and NUPA (Nested Universal Primer A) were used, with the products of the first amplification as templates. The first and nested PCRs were carried out under the same conditions: 5 cycles of $30 \mathrm{~s}$ at $94^{\circ} \mathrm{C}, 2 \mathrm{~min}$ at $60^{\circ} ; 5$ cycles of $30 \mathrm{~s}$ at $94^{\circ} \mathrm{C}, 30 \mathrm{~s}$ at $55^{\circ} \mathrm{C}$, and $2 \mathrm{~min}$ at $72^{\circ} \mathrm{C}$; and 25 cycles of $30 \mathrm{~s}$ at $94^{\circ} \mathrm{C}, 30 \mathrm{~s}$ at $55^{\circ} \mathrm{C}$, and $1 \mathrm{~min}$ at $72^{\circ} \mathrm{C}$. The $3^{\prime}$ RACE and nested $5^{\prime}$ RACE products were purified and cloned into the pMD18-T vector (TaKaRa, China), followed by sequencing.

By aligning and assembling the core fragment, 5' RACE, and 3' RACE products, the full-length cDNA of CsEIN3 was deduced and confirmed by RT-PCR using the primers CsEIN3-Full-Fw and CsEIN3-Full-Re.

\section{Sequence analysis and 3-D modeling}

The ORF prediction was carried out with Genscan (http://genes.mit.edu/GENSCAN. $\mathrm{html})$. The similar sequences of other species were obtained by using the BLASTP tool at the NCBI web site (http://www.ncbi.nlm.nih.gov/BLAST/). Multiple sequences alignment was performed using ClustalX 2.0 and processed by GeneDoc (http://www.nrbsc.org/gfx/genedoc/ index.html). MEGA (Molecular Evolutionary Genetics Analysis, version 2.1) was subsequently used to construct the phylogenetic tree by applying the neighbor-joining method. Homology modeling was performed with the SWISS-MODEL program on the ExPASy web server (http://www.expasy.org/) (Arnold et al., 2006).

\section{Expression analysis by quantitative real-time PCR}

Quantitative real-time PCR (Q-PCR) was performed to investigate the expression level of CsEIN3 mRNA in different plant organs including roots, stem, leaves, shoot apices, and mature male and female flowers, and in different developmental stages of both male and female flowers, using the SYBR Green method and FTC-3000TM System Real-time Quantitative Thermal Cycler (Funglyn Biotech, Canada).

The cDNA for Q-PCR was synthesized from total RNA extracted from various tissues (three replicates per tissue) using the ReverTra Ace ${ }^{\circledR}$ qPCR RT Kit (Toyobo, China), according to the kit instructions, with oligo(dT) primers. The total RNA used for reverse transcription was incubated with DNase I (Fermentas, China) for $30 \mathrm{~min}$ at $37^{\circ} \mathrm{C}$ to remove the DNA contamination. The primer for Q-PCR was designed using Primer Premier 5.0, and the amplification efficiency was evaluated via standard curve analysis.

The Q-PCR reactions were performed on a 96-well plate, in triplicate, with each reaction consisting of a $25-\mu \mathrm{L}$ mixture that contained $12.5 \mu \mathrm{L} 2 \mathrm{X}$ UltraSYBR Mixture (CWBiotech), $0.4 \mu \mathrm{M}$ each primer, and $20 \mathrm{ng}$ cDNA. The amplification conditions were as follows: $10 \mathrm{~min}$ at $95^{\circ} \mathrm{C}$ to activate the DNA polymerase, followed by 40 cycles of $15 \mathrm{~s}$ at $95^{\circ} \mathrm{C}$ and 1 min at $60^{\circ} \mathrm{C}$. A dissociation curve was performed to ensure the validity of each specific PCR product. The Q-PCR was repeated three times.

The relative expressions of the CSEIN3 genes were calculated based on the $2^{-\Delta \Delta C t}$ method (Livak and Schmittgen, 2001), using the CsActin 3 gene as an internal standard. 


\section{RESULTS}

\section{Cloning of the full-length cDNA of CsEIN3}

Using the above-mentioned methods, the full-length cDNA designated as CsEIN3 was obtained. CsEIN3 was 2560 bp long, including a 194 bp 5'-untranslated region (UTR), a 458 bp 3'-UTR, a 1908 bp ORF encoding 635 amino acids residues, and a poly(A) tail of $23 \mathrm{bp}$. In addition, a potential polyadenylation signal (AAATAA) was found $10 \mathrm{bp}$ upstream from the poly(A) stretch (Figure 1).

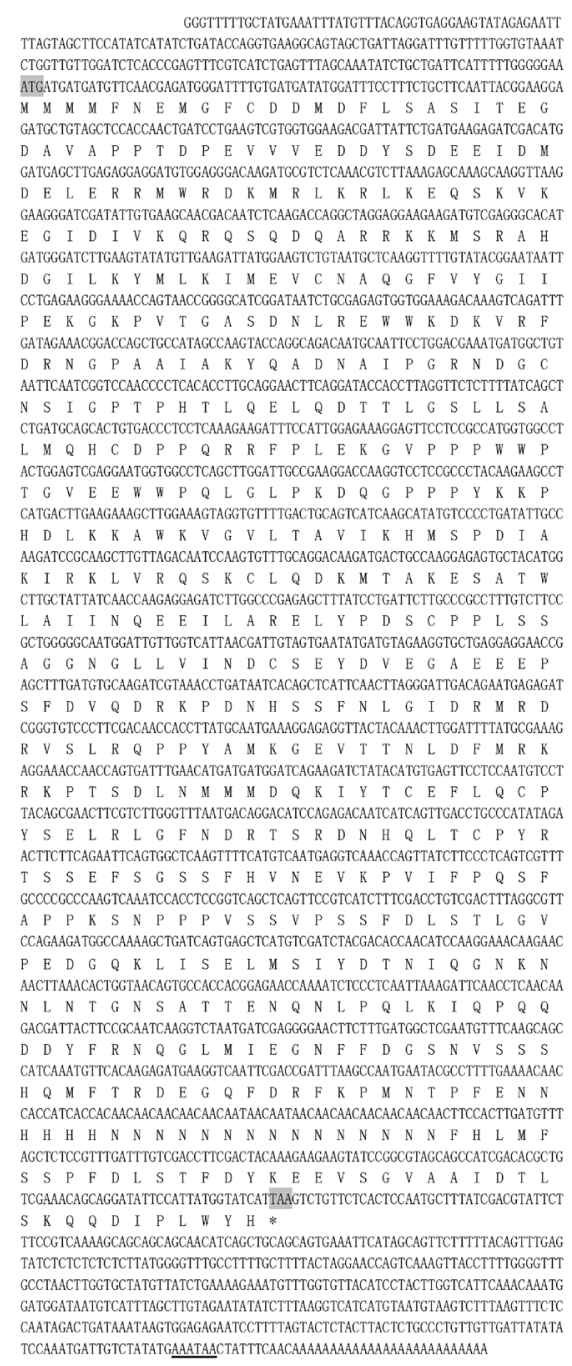

Figure 1. Full-length cDNA and deduced amino acid sequences of CsEIN3. The start codon (ATG) is shaded. The stop codon (TAA) is also shaded and marked by an asterisk. The putative polyadenylation signal (AAATAA) is underlined. 


\section{Multiple sequence alignment and phylogenetic analysis}

Multiple amino acid sequence alignments showed that CsEIN3 had very high homology with its counterparts from other plant species (Figure 2). CsEIN3 had identities of 67, 64, 70 , and $62 \%$ to NtEIL1, SIEIL1, VrEIL2, and AtEIN3, respectively, indicating that the protein structure and functional manner were strongly conserved. Sequence alignment revealed that there was high homology in the N-terminus, including the DNA-binding domains that consisted of five conserved $\alpha$-helices. The lysine $245(\mathrm{~K})$ located in $\alpha$-helices-3, the key amino acid residue responsible for the DNA-binding and signal transduction activities, was found in CsEIN3 (Yamasaki et al., 2005).

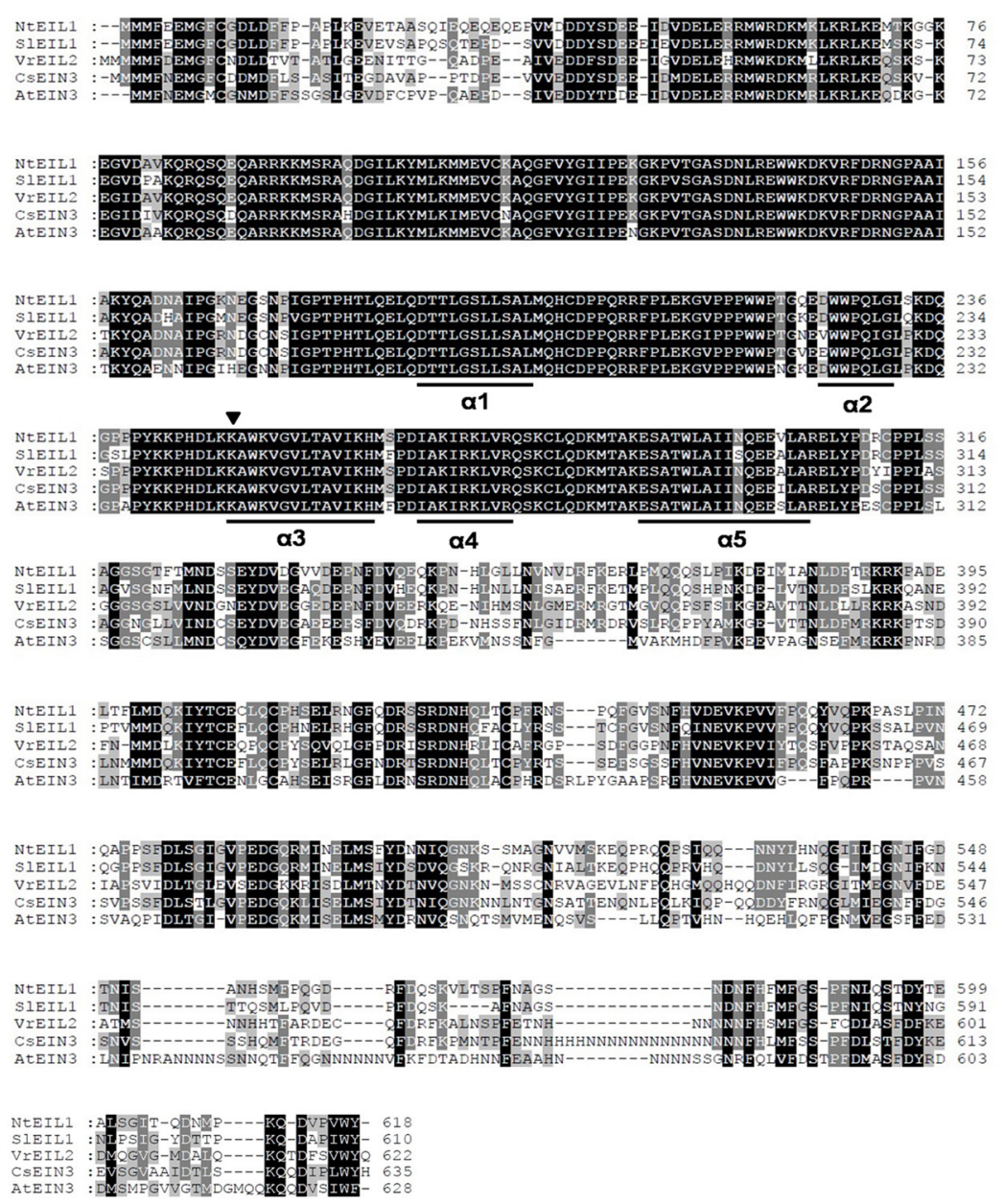

Figure 2. Multiple alignments of deduced amino acid sequence of CsEIN3 with other known plant EIN3/EIL genes. Five EIN3/EIL genes from Nicotiana tabacum (NtEIL1, AAP03997), Solanum lycopersicum (SIEIL1, AAK58857), Vigna radiata (VrEIL2, AAL76271), Cucumis sativus (CsEIN3, AFK80347), Arabidopsis thaliana (AtEIN3, AEE76421) were used for multiple sequence alignment. Five $\alpha$-helices are underlined, and the key amino acid residue responsible for the DNA-binding and signal transduction activities are indicated with black triangles. 
To understand the evolutionary relationships among CSEIN3 and other plant EIN3/ EILs, a phylogenetic tree was constructed based on the amino acid sequences of plant EIN3/ EILs (Figure 3). It was revealed that plant EIN3/EILs were derived from a common ancestor during evolution, and CsEIN3 was grouped into a cluster along with melon, belonging to the Cucurbitaceae and paralleling their evolutionary relationships.

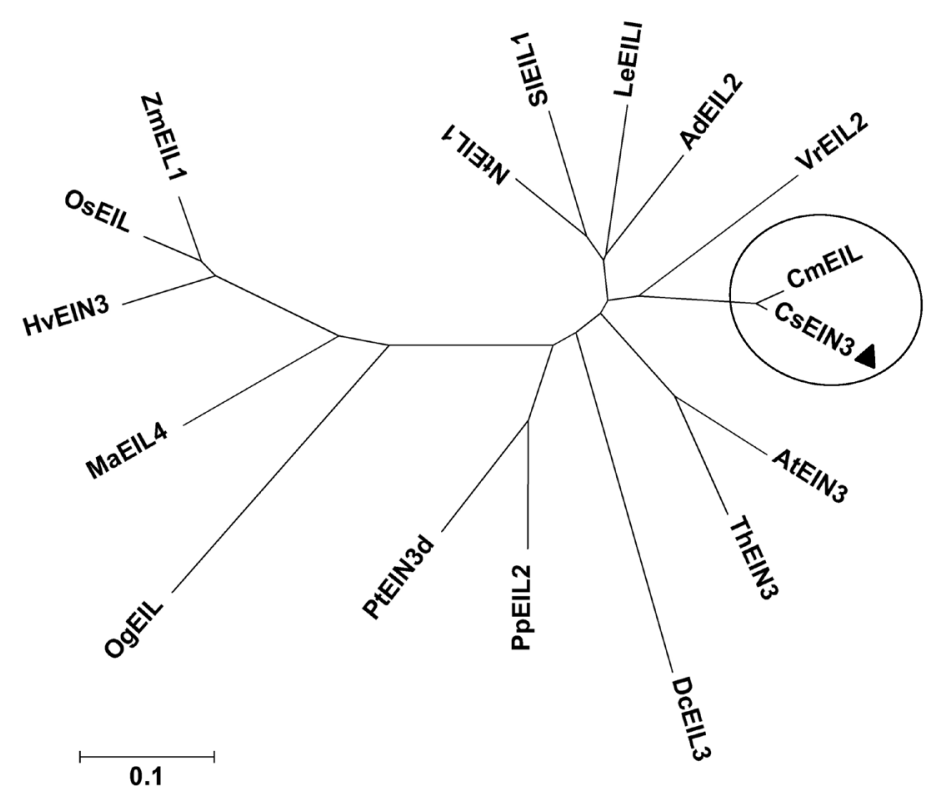

Figure 3. Phylogenetic relationships of known EIN3/EILs in plants. Amino acid sequences used for alignment include CmEIL (Cucumis melo, BAB64344), VrEIL2 (Vigna radiata, AAL76271), NtEIL1 (Nicotiana tabacum, AAP03997), SIEIL1 (Solanum lycopersicum, AAK58857), AdEIL2 (Actinidia deliciosa, ACJ70675), LeEIL1 (Lithospermum erythrorhizon, ACP56697), AtEIN3 (Arabidopsis thaliana, AEE76421), PpEIL2 (Prunus persica, ABK35086), DcEIL3 (Dianthus caryophyllus, AAV68141), MaEIL4 (Musa acuminate, ABG89103), OsEIL (Oryza sativa, BAB78462), ZmEIL1 (Zea mays, NP 001152035), OgEIL (Oncidium Gower Ramsey, AEK84144), PtEIN3d (Populus trichocarpa, XP_002310961), ThEIN3 (Thellungiella halophila, BAJ33951), HvEIN3 (Hordeum vulgare, BAJ95094). The scale bar represents the estimated evolutionary distance as 0.1 amino acid substitutions per site. CsEIN3 (indicatied with black triangles) is grouped together with counterparts from melon (CmEIL), as indicated by the ellipse.

\section{3-D modeling of CsEIN3 protein}

To better understand the protein character of CsEIN3, a 3-D model of the CsEIN3 protein was established by SWISS-MODEL using the homology-modeling method. The template for modeling was the crystal structure of the EIL3 protein from Arabidopsis thaliana (PDB: 1wijA; Kazuhiko et al, 2005). We obtained a 3-D structure of the CsEIN3 protein fragment (178 to 301 aa) containing the DNA-binding domain. The result showed that five $\alpha$-helices formed a V-shaped cleft, and the key lysine 245 (Lys245) responsible for the DNA-binding and signal transduction activities was located at a corresponding position similar to the template of AtEIL3 (Figure 4). 

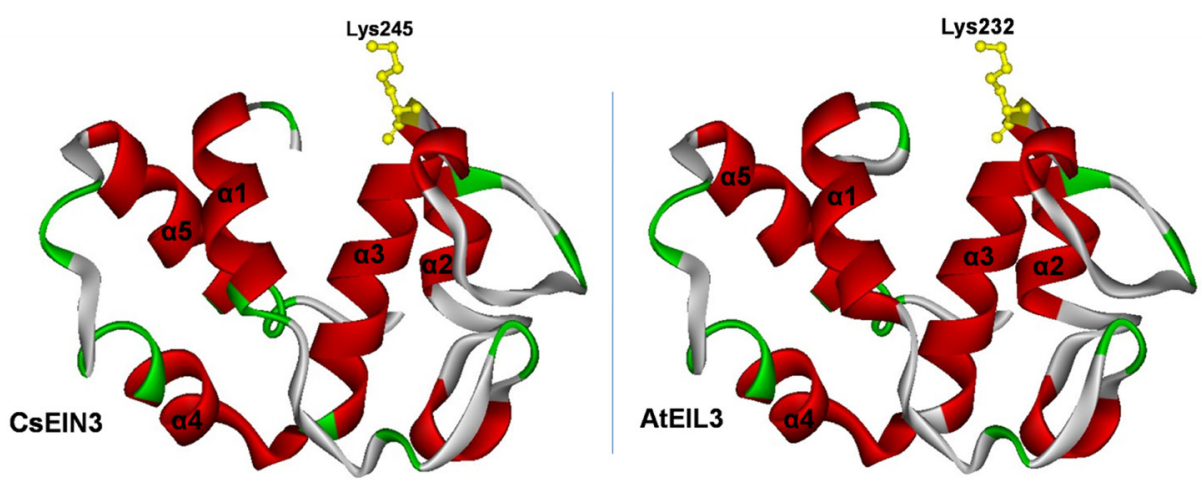

Figure 4. Three-dimensional structure of CsEIN3 fragment (178-301 aa) contains DNA-binding domain (DBD). Five $\alpha$-helices $(\alpha 1-\alpha 5)$ are indicated by red helices, the key amino acid residue (lysine) responsible for the DNAbinding and signal transduction activities are indicated with yellow ball.

\section{Expression patterns of $C s E I N 3$ in different organs}

To help us elucidate the role of CSEIN3 in cucumber, the gene expression was analyzed in different plant organs by quantitative real-time RT-PCR, including roots, stems, leaves, shoot apices, and mature male and mature female flowers. The results showed that CSEIN3 was expressed in all the organs tested. The highest expression of CSEIN3 was detected in mature male flowers, mature female flowers, and stems. With regard to mature flowers, the expression level of CsEIN3 in male flowers was higher than in female flowers (Figure 5).

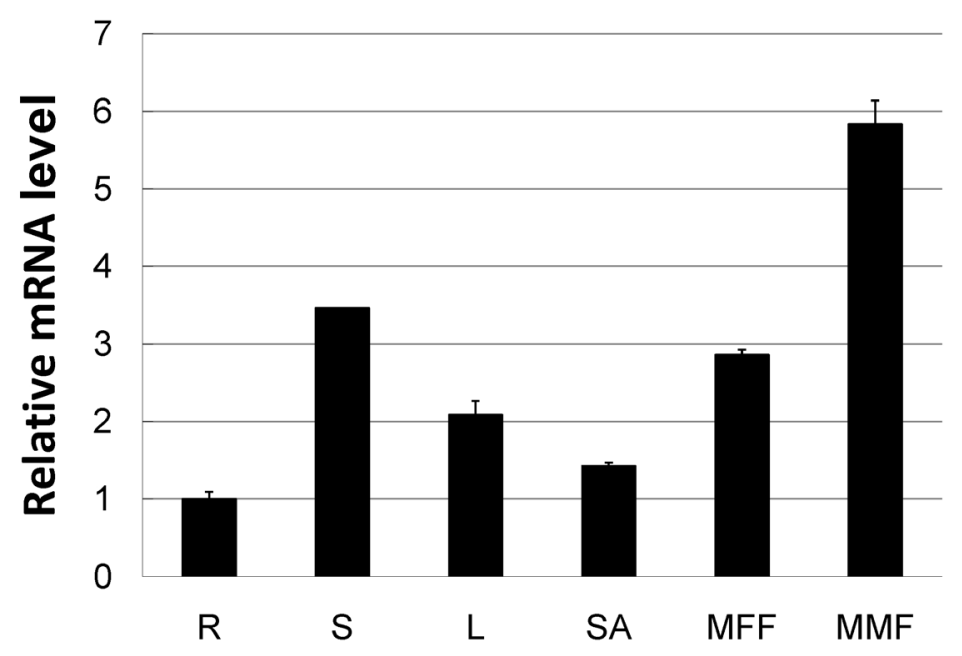

Figure 5. Expression analysis of CsEIN3 in different organs of cucumber. Three biological replicate experiments are reported as means $\pm \mathrm{SD}$. $\mathrm{R}=$ roots; $\mathrm{S}=$ stem; $\mathrm{L}=$ leaves; $\mathrm{SA}=$ shoot apices; $\mathrm{MMF}=$ mature male flowers; $\mathrm{MFF}$ $=$ mature female flowers. 


\section{Expression patterns of CsEIN3 in different developmental stages of flowers}

To further understand whether CsEIN3 was indeed involved in flower development, we preliminarily explored the expression patterns of CSEIN3 in six different stages (from MF1/FF1 to MMF/MFF) throughout the development of female and male flowers (Figure 6A shows FF1-FF5 in females and MF1-MF5 in males). The results showed that accumulation of CSEIN3 mRNA increased during both male and female flowers development and maturation on the whole, although it declined slightly at stages FF4 and FF5 in female flowers. At the early stages of flower development (MF1/FF1 and MF2/FF2), the expression of CsEIN3 was relatively low (Figure 6B).
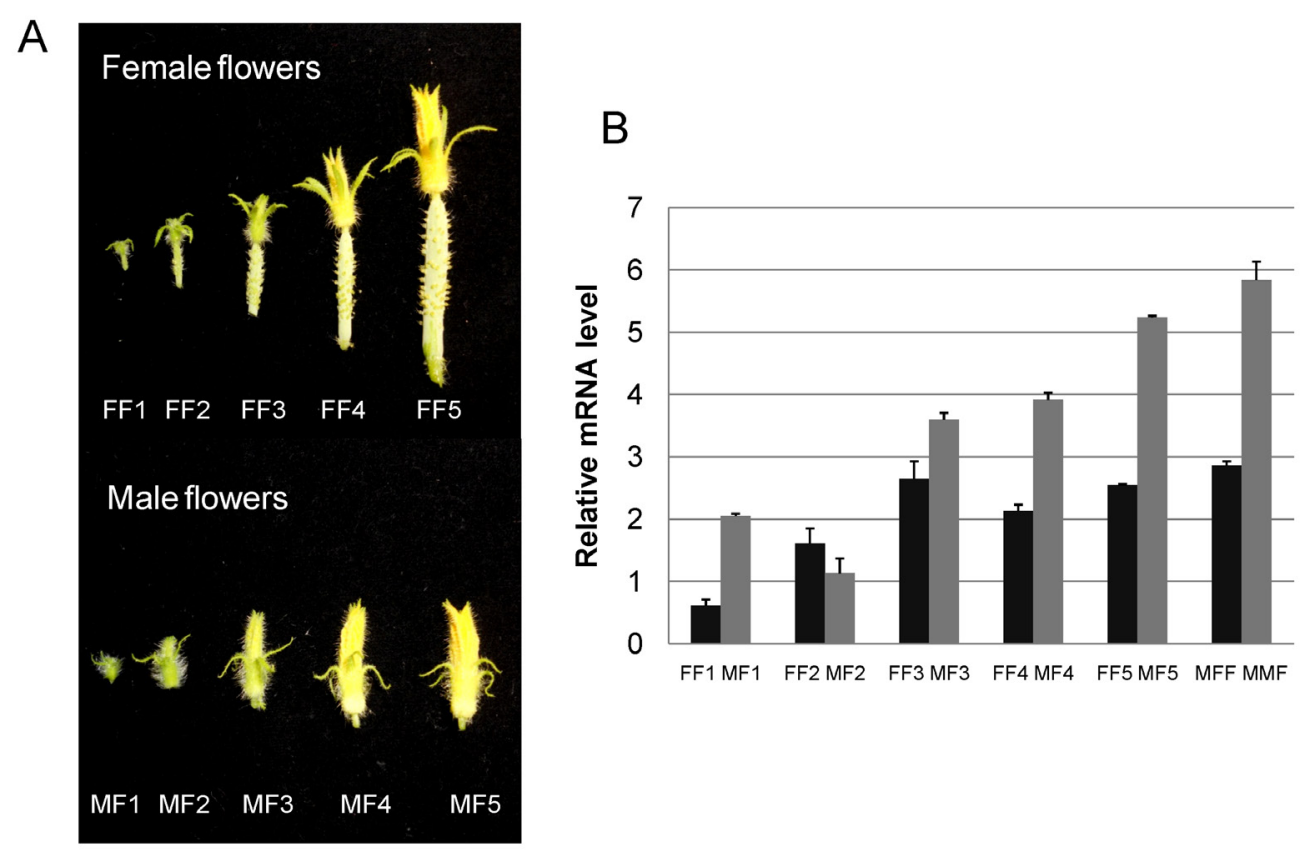

Figure 6. A. Morphology of male (MF) and female (FF) flowers at developmental stages, which were separated on the basis of the corolla length. MF1/FF1 $=2.5 \pm 1 \mathrm{~mm}, \mathrm{MF} 2 / \mathrm{FF} 2=5 \pm 1 \mathrm{~mm}, \mathrm{MF} 3 / \mathrm{FF} 3=9 \pm 2 \mathrm{~mm}, \mathrm{MF} 4 / \mathrm{FF} 4$ $=15 \pm 2 \mathrm{~mm}, \mathrm{MF} 5 / \mathrm{FF} 5=20 \pm 2 \mathrm{~mm}$. B. Relative expression of CsEIN3 in FF and MF at different developmental stages. The three biological replicate experiments are reported as means $\pm \mathrm{SD}$.

\section{DISCUSSION}

Cucumber is considered a model plant for studying flower development, particularly the ethylene promotion of female flowering during unisexual flower development. Therefore, the isolation and characterization of relevant components are the foundation for study of the molecular mechanism of the cucumber ethylene signaling pathway. EIN3/EIL proteins, as key components in the ethylene signaling pathway, have still not been isolated from cucumber, thus hampering the study on their biological functions in cucumber.

In the present paper, we first reported the full-length cDNA sequence of the EIN3 gene 
in cucumber. The ORF of CsEIN3 was $1908 \mathrm{bp}$ in length, encoding a putative polypeptide of 635 amino acids. Initially, our experimental results were not in accordance with the tentative consensus sequence of csa012885 from the Cucumber Genome Database, of which the ORF was $1395 \mathrm{bp}$ in length and encoding 464 amino acids. In order to clarify this inconsistent result, we aligned these two sequences and found that csa012885 lacked two fragments. To examine whether this situation was caused by a gap of genome sequencing, we next downloaded the genomic sequence corresponding to csa012885 from the database and amplified the genomic sequence of CSEIN3, respectively. Interestingly, the genomic sequence of CsEIN3 did not contain an intron of which the length was also $1908 \mathrm{bp}$, like the cDNA sequence (data not shown). Based on this clue, we obtained a putative ORF of 622 amino acids, using the genomic sequence of csa012885, which was $1869 \mathrm{bp}$ in length by ORF prediction; obviously, it also did not contain the intron. Subsequently, we aligned these two amino acid sequences and found that they had an identical sequence, except that there were serial 15 asparagines (Asn, $\mathrm{N}$ ) at the C-terminus, whereas there were merely 3 asparagines in csa012885. Furthermore, we aligned the 1395 and 1869 bp sequences and found that the differential sequence conformed to the "GT-AG" rule. Therefore, the inaccuracy for the csa012885 sequence provided by the Cucumber Genome Database is most likely caused by the gene prediction algorithm that identified this fragment as an intron, which does not appear in csa012885. As for the number of serial asparagines $(\mathrm{N})$ at the C-terminus, it is probably due to differences in cultivars. It is noteworthy that CmEIL1, which is the counterpart of CsEIN3 in melon, also lacked the intron when we compared its genomic sequence and mRNA sequence (data not shown).

Bioinformatics analysis showed that CsEIN3 possessed properties of EIN3/EILs by virtue of its highly conserved amino acid sequence and the strongly similar structure of the functional domain when compared with other plant EIN3/EILs. Specifically, CsEIN3 showed high homology with its counterpart (CmEIL1) in melon and was grouped into the same cluster together with melon, which belongs to the same genus as cucumber. Subcellular localization prediction by WoLF PSORT (Horton et al., 2007) indicated that CsEIN3 was localized in the nucleus (data not shown). This result was consistent with EIN3/EILs being nuclear transcription factors.

The previous studies have indicated that EIN3/EILs are expressed in almost all examined tissues; however, the expression pattern showed high diversity among different EIN3/ EIL family members. For instance, the tomato EIN3/EIL family consists of four members (LeEIL1, LeEIL2, LeEIL3, and LeEIL4), all four of which were detected in all tissues examined. Interestingly, a high expression level of LeEIL4 was detected in fruits and increased during fruit ripening, whereas the other three members were highly expressed in roots, stems, and flowers (Yokotani et al., 2003). In this study, we found that CsEIN3 was expressed in all the plant organs examined including roots, stems, leaves, shoot apices, and male and female flowers, although the expression level was somewhat higher in flowers and stems than in the other organs. It is noteworthy that the accumulation of CsEIN3 mRNA increased during flower development and maturation in both male and female flowers. This increase might be involved in the regulation of flower development and maturation in response to ethylene, consistent with a previous report (Iordachescu and Verlinden, 2005).

In summary, we have successfully cloned the full-length cDNA of the EIN3 gene from cucumber and investigated its expression pattern in different plant organs and different developmental stages of flowers. This study will lay the foundation for further investigation into the role of EIN3/EIL protein factors in relevant biological processes of cucumber and the molecular mechanism of the cucumber ethylene signaling pathway. 


\section{ACKNOWLEDGMENTS}

Research supported by the National Program on Key Basic Research Projects (The "973" Program: \#2012CB113900) and the National Natural Science Foundation of China (\#31071081 and \#31272185).

\section{REFERENCES}

An F, Zhao Q, Ji Y, Li W, et al. (2010). Ethylene-induced stabilization of ETHYLENE INSENSITIVE3 and EIN3-LIKE1 is mediated by proteasomal degradation of EIN3 binding F-box 1 and 2 that requires EIN2 in Arabidopsis. Plant. Cell 22: 2384-2401.

An F, Zhang X, Zhu Z, Ji Y, et al. (2012). Coordinated regulation of apical hook development by gibberellins and ethylene in etiolated Arabidopsis seedlings. Cell Res. 22: 915-927.

Ando S, Sato Y, Kamachi S and Sakai S (2001). Isolation of a MADS-box gene (ERAF17) and correlation of its expression with the induction of formation of female flowers by ethylene in cucumber plants (Cucumis sativus L.). Planta 213: 943-952.

Arnold K, Bordoli L, Kopp J and Schwede T (2006). The SWISS-MODEL workspace: a web-based environment for protein structure homology modelling. Bioinformatics 22: 195-201.

Bauer P and Blondet E (2011). Transcriptome analysis of ein3 eill mutants in response to iron deficiency. Plant Signal. Behav. 6: 1669-1671.

Cao WH, Liu J, He XJ, Mu RL, et al. (2007). Modulation of ethylene responses affects plant salt-stress responses. Plant Physiol. 143: 707-719.

Chao Q, Rothenberg M, Solano R, Roman G, et al. (1997). Activation of the ethylene gas response pathway in Arabidopsis by the nuclear protein ETHYLENE-INSENSITIVE3 and related proteins. Cell 89: 1133-1144.

Chen G, Alexander L and Grierson D (2004). Constitutive expression of EIL-like transcription factor partially restores ripening in the ethylene-insensitive Nr tomato mutant. J. Exp. Bot. 55: 1491-1497.

Chen H, Xue L, Chintamanani S, Germain H, et al. (2009). ETHYLENE INSENSITIVE3 and ETHYLENE INSENSITIVE3-LIKE1 repress SALICYLIC ACID INDUCTION DEFICIENT2 expression to negatively regulate plant innate immunity in Arabidopsis. Plant Cell 21: 2527-2540.

De Paepe A, Vuylsteke M, Van Hummelen P, Zabeau M, et al. (2004). Transcriptional profiling by cDNA-AFLP and microarray analysis reveals novel insights into the early response to ethylene in Arabidopsis. Plant J. 39: 537-559.

Guo H and Ecker JR (2004). The ethylene signaling pathway: new insights. Curr. Opin. Plant Biol. 7: 40-49.

Hibi T, Kosugi S, Iwai T, Kawata M, et al. (2007). Involvement of EIN3 homologues in basic PR gene expression and flower development in tobacco plants. J. Exp. Bot. 58: 3671-3678.

Horton P, Park KJ, Obayashi T, Fujita N, et al. (2007). WoLF PSORT: protein localization predictor. Nucleic Acids Res. 35: W585-W587.

Huang S, Li R, Zhang Z, Li L, et al. (2009). The genome of the cucumber, Cucumis sativus L. Nat. Genet. 41: 1275-1281. Iordachescu M and Verlinden S (2005). Transcriptional regulation of three EIN3-like genes of carnation (Dianthus caryophyllus L. cv. Improved White Sim) during flower development and upon wounding, pollination, and ethylene exposure. J. Exp. Bot. 56: 2011-2018.

Knopf RR and Trebitsh T (2006). The female-specific Cs-ACS1G gene of cucumber. A case of gene duplication and recombination between the non-sex-specific 1-aminocyclopropane-1-carboxylate synthase gene and a branchedchain amino acid transaminase gene. Plant Cell Physiol. 47: 1217-1228.

Lee JH and Kim WT (2003). Molecular and biochemical characterization of VR-EILs encoding mung bean ETHYLENE INSENSITIVE3-LIKE proteins. Plant Physiol. 132: 1475-1488.

Li Z, Huang S, Liu S, Pan J, et al. (2009). Molecular isolation of the M gene suggests that a conserved-residue conversion induces the formation of bisexual flowers in cucumber plants. Genetics 182: 1381-1385.

Lin Z, Zhong S and Grierson D (2009). Recent advances in ethylene research. J. Exp. Bot. 60: 3311-3336.

Liu S, Xu L, Jia Z, Xu Y, et al. (2008). Genetic association of ETHYLENE-INSENSITIVE3-like sequence with the sexdetermining M locus in cucumber (Cucumis sativus L.). Theor. Appl. Genet. 117: 927-933.

Livak KJ and Schmittgen TD (2001). Analysis of relative gene expression data using real-time quantitative PCR and the 2(-Delta Delta C(T)) Method. Methods 25: 402-408.

Maruyama-Nakashita A, Nakamura Y, Tohge T, Saito K, et al. (2006). Arabidopsis SLIM1 is a central transcriptional regulator of plant sulfur response and metabolism. Plant Cell 18: 3235-3251. 
Neljubov DN (1901). Über die horizontale Nutation der Stengel von Pisum sativum und einiger anderen Pflanzen. Beih. Bot. Centralbh. 10: 129-139.

Parra-Lobato MC and Gomez-Jimenez MC (2011). Polyamine-induced modulation of genes involved in ethylene biosynthesis and signalling pathways and nitric oxide production during olive mature fruit abscission. J. Exp. Bot. 62: 4447-4465.

Rudich J, Halevy AH and Kedar N (1972). Ethylene evolution from cucumber plants as related to sex expression. Plant Physiol. 49: 998-999.

Shi Y, Tian S, Hou L, Huang X, et al. (2012). Ethylene signaling negatively regulates freezing tolerance by repressing expression of CBF and type-A ARR genes in Arabidopsis. Plant Cell 24: 2578-2595.

Tieman DM, Ciardi JA, Taylor MG and Klee HJ (2001). Members of the tomato LeEIL (EIN3-like) gene family are functionally redundant and regulate ethylene responses throughout plant development. Plant J. 26: 47-58.

Yamasaki K, Kigawa T, Inoue M, Yamasaki T, et al. (2005). Solution structure of the major DNA-binding domain of Arabidopsis thaliana ethylene-insensitive3-like3. J. Mol. Biol. 348: 253-264.

Yamasaki S, Fujii N and Takahashi H (2000). The ethylene-regulated expression of CS-ETR2 and CS-ERS genes in cucumber plants and their possible involvement with sex expression in flowers. Plant Cell Physiol. 41: 608-616.

Yanagisawa S, Yoo SD and Sheen J (2003). Differential regulation of EIN3 stability by glucose and ethylene signalling in plants. Nature 425: 521-525.

Yokotani N, Tamura S, Nakano R, Inaba A, et al. (2003). Characterization of a novel tomato EIN3-like gene (LeEIL4). J. Exp. Bot. 54: 2775-2776.

Zhang L, Li Z, Quan R, Li G, et al. (2011). An AP2 domain-containing gene, ESE1, targeted by the ethylene signaling component EIN3 is important for the salt response in Arabidopsis. Plant Physiol. 157: 854-865.

Zhu Z, An F, Feng Y, Li P, et al. (2011). Derepression of ethylene-stabilized transcription factors (EIN3/EIL1) mediates jasmonate and ethylene signaling synergy in Arabidopsis. Proc. Natl. Acad. Sci. U. S. A. 108: 12539-12544. 